\title{
Can computer mice be used as low-cost devices for the acquisition of planar human movement velocity signals?
}

\author{
Christian O'Reilly • Réjean Plamondon
}

Published online: 16 November 2010

(C) Psychonomic Society, Inc. 2010

\begin{abstract}
The main goal of this work is to determine whether a computer mouse can be used as a low-cost device for the acquisition of two-dimensional human movement velocity signals in the context of psychophysical studies and biomedical applications. A comprehensive overview of the related literature is presented, and the problem of characterizing mouse movement acquisition is analyzed and discussed. Then, the quality of velocity signals acquired with this kind of device is measured on horizontal oscillatory movements by comparing the mouse data to the signals acquired simultaneously by a video motion tracking system and a digitizing tablet. A synthesis of the information gathered in this work indicates that the computer mouse can be used for the reliable acquisition of biosignals in the context of human movement studies, particularly for many applications dealing with the velocity of the end effector of the upper limb. This paper concludes by discussing the possibilities and limitations of such use.
\end{abstract}

Keywords Data acquisition $\cdot$ Pointing device $\cdot$ Motion tracking $\cdot$ Computer mouse $\cdot$ Human movement $\cdot$ Biosignal

\section{Introduction}

As the computer mouse is standard acquisition equipment available on almost all modern computers, it is tempting to use it to record velocity signals in various applications or in laboratory experiments as an alternative to more expensive equipment. In this paper, we study the possibility of using

\author{
C. O'Reilly $(\square) \cdot$ R. Plamondon \\ Département de Génie Électrique, École Polytechnique de \\ Montréal, Laboratoire Scribens, \\ C.P. 6079, Succursale Centre-Ville, \\ Montréal, QC H3C 3A7, Canada \\ e-mail: christian.oreilly@polymtl.ca \\ R. Plamondon \\ e-mail: rejean.plamondon@polymtl.ca
}

this device as a cheap but precise and reliable acquisition tool for studying the velocity of human arm motion. Many disciplines could benefit from using a computer mouse for such a purpose, including biomedical engineering (O'Reilly, Plamondon, Lebrun, Clément, \& Mathieu, 2009), experimental psychology (Brenner \& Smeets, 2003; Jansen, Blackwell, \& Marriott, 2003; Morein-Zamir, Chua, Franks, Nagelkerke, \& Kingstone, 2006; Nagel, Kopiez, Grewe, \& Altenmuller, 2007; Thompson, McConnell, Slocum, \& Bohan, 2007; Walker, Meyer, \& Smelcer, 1993), the neurosciences (Ogawa, Inui, \& Sugio, 2007; Roby-Brami \& Burnod, 1995; Young, Pratt, \& Chau, 2008), rehabilitation engineering (O'Reilly \& Plamondon, 2009), ergonomics (Phillips \& Triggs, 2001), and so on.

In Sect. "An overview of previous studies", we present the acquisition devices most commonly used for recording human upper arm movements, and surveys previous work published on the use of mice as movement sensors. In Sect. "Characterization of computer mouse acquisition", we specify more precisely what component of the measurement error this study aims to analyze. In Sect. "Experimentation", experimental results on the characterization of mouse signal quality are given. In Sect. "Discussion and recommendations", these results are discussed and some recommended guidelines for reliable acquisitions with computer mice are formulated. Finally, in Sect. "Conclusions", the main contributions of this paper are summarized, and a positive answer to the initial research question is provided.

\section{An overview of previous studies}

Movement acquisition devices for human motion tracking

This overview of the acquisition devices commonly used in human movement analysis is restricted to those that are of interest for comparison with a computer mouse. Thus, 
systems for other types of movement, such as tremors, eye saccades, mandible movements, and gait are not addressed here. We have limited this study to devices that could eventually be used to track human upper limb movements. Some references are given as examples for each type of device, but this is by no means an exhaustive list. Readers interested in a recent and more comprehensive discussion on this topic can consult (Zhou \& Hu, 2008).

Four main types of device have been widely used for human movement acquisition. Biomechanical studies often use camera and marker systems (Ehara et al., 1997; Ehara, Fujimoto, Miyazaki, Tanaka, \& Yamamoto, 1995; Everaert, Spaepen, Wouters, Stappaerts, \& Oostendorp, 1999; Hassan, Jenkyn, \& Dunning, 2007; Kolahi et al., 2007; Richards, 1999). These have good resolution and are useful when the 3D kinematics of multiple body segments is needed, such as in joint coordination analysis. However, they are rather expensive, owing to the use of many cameras - generally at least six, and often more than ten. They also require the placement of markers on the subject, which can be a relatively long and delicate process that can be considered somewhat invasive from the subject's point of view. This kind of system is also subject to marker occlusion.

Less expensive than markers and cameras are electromagnetic tracking systems (Biryukova, Roby-Brami, Frolov, \& Mokhtari, 2000; Hassan et al., 2007; Mills, Morrison, Lloyd, \& Barrett, 2007; Richards, 1999). These offer good resolution, but electromagnetic interference from nearby metal objects has to be carefully considered.

Inertial sensor-based systems have also been proposed for movement recording (Mayagoitia, Nene, \& Veltink, 2002; Picerno, Cereatti, \& Cappozzo, 2008; Plamondon et al., 2007; Zhou, Hu, Harris, \& Hammerton, 2006; Zhou, Stone, Hu, \& Harris, 2008). These have the advantage of being wearable, freeing the subject from the constraints of executing movements within a defined volume, as is the case with the systems mentioned previously (i.e., markers in magnetic or optical systems are wearable, but the acquisition system is not, which requires that the subject constrain his movements to the volume covered by the acquisition equipment).

Digitizing tablets, which are commonly adopted for online handwriting and signatures studies (Impedovo \& Pirlo, 2008; Leclerc \& Plamondon, 1994; Plamondon \& Lorette, 1989; Plamondon \& Srihari, 2000), and increasingly used by biomechanical or biomedical practitioners in the last decade (see (Gilboa, Josman, Fattal-Valevski, Toledano-Alhadef, \& Rosenblum, 2010; Lange et al., 2007; Swett, ContrerasVidal, Birn, \& Braun, 2010; Van Gemmert, Teulings, \& Stelmach, 2001) for some typical examples), give the reliable and accurate movement acquisition of a pen tip. This tool has been used for studies dealing with 2D movements of limited amplitude.
These four types of acquisition device record positional signals, while the standard computer mouse records position change, which is closer to velocity ${ }^{1}$ than position. Therefore, in this study, the mouse has been tested as a possible low-cost alternative to the other four types of acquisition device only for velocity analysis, since it is, by design, vulnerable to position error accumulation. ${ }^{2}$

Computer mice as movement acquisition devices

Although computer mice have some important limitations (e.g., they record the planar position changes of an end effector rather than the three-dimensional positions of multiple effectors), they also have some notable advantages, such as low cost, compactness, and availability.

Some work has been published on the utilization of an optical mouse for movement acquisition in an industrial context (Kok-Meng \& Debao, 2004; Ng \& Cheong, 2004), in biometric systems (Ahmed \& Traore, 2007), and as a robot odometer (Bonarini, Matteucci, \& Restelli, 2005; Cooney, Xu, \& Bright, 2004; Palacin, Valganon, \& Pernia, 2006; Santos, Silva, \& Almeida, 2002; Singh \& Waldron, 2004). It has also been used as a low-cost acquisition tool for student laboratories (Daya, Jinghua, Haihong, \& Qionglin, 2007; Ng \& Ang, 2005; Ng \& Carne, 2007; Ochoa \& Kolp, 1997).

Some human movement experiments (Brenner \& Smeets, 2003; Faustina, Simeon, Patrick, \& John, 2004; Jansen et al., 2003; Kisacanin, Agarwal, Taber, \& Hier, 2000; Morein-Zamir et al., 2006; Nagel et al., 2007; Nijhof, 2003; Ogawa et al., 2007; Phillips \& Triggs, 2001; Pratt \& Turk-Browne, 2003; Roby-Brami \& Burnod, 1995; Slijper, Richter, Over, Smeets, \& Frens, 2009; Thompson et al., 2007; Walker et al., 1993) have used computer mice as well, but without detailed analysis of signal acquisition reliability. In these studies, mouse signals seem to have been considered acceptable a priori. This may be suitable for some kind of studies (e.g., studies concerned with the inter-class versus intra-class variability between two tests using the same acquisition device, investigations of phenomena related to human motion that does not require high temporal or spatial accuracy). However, it is not for studies dealing, for example, with the precise characterization of human movement velocity, such as handwriting

\footnotetext{
${ }^{1}$ Although what a computer mouse records is close to velocity, it does not in fact record velocity, since the reported position changes are not multiplied by the sampling frequency. Moreover, it was not clear before this study whether or not good quality velocity signals could be obtained by making this multiplication a posteriori, because the sampling frequency varies with time and its value is not precisely known.

${ }^{2}$ To obtain position, mouse signals would have to be integrated, and this operation is known to accumulate errors.
} 
analysis (Djioua \& Plamondon, 2009b), stroke prevention (O'Reilly \& Plamondon, 2010; O'Reilly et al., 2009), or rehabilitation (Dipietro, Krebs, Fasoli, Volpe, \& Hogan, 2009; Rohrer et al., 2004).

Studies evaluating mouse timing characteristics

A few papers were published in the early 1990s on using the computer mouse as a low-cost response box in experimental psychology. For such an application, (Segalowitz \& Graves, 1990) reported delays of $31 \pm 2 \mathrm{~ms}$ when the mouse was at rest, and, most importantly, a longer and more variable delay of $45 \pm 15 \mathrm{~ms}$ when it was moving. (Crosbie, 1990) reported a mean delay of $35.15 \mathrm{~ms}$ with a standard deviation of $3.54 \mathrm{~ms}$ for button presses on a fixed serial mouse, and a mean delay of $4.1 \mathrm{~ms}$ with a standard deviation of 1.34 with a fixed bus mouse. Beringer (1992) reported similar results, but with some new insights concerning the inter-key interval properties of button press acquisition.

The information contained in these earlier studies might seem a little obsolete for today's researchers, who rely on very different hardware and software. To address this shortcoming, (Plant, Hammond, \& Whitehouse, 2003) published an update on the subject. Studying various mouse models using different protocols (serial, PS/2, USB), they reported a delay of about $31 \mathrm{~ms}$ related to the internal mouse circuitry, with an additional delay of about $5 \mathrm{~ms}$ related to the computeracquisition process. The total delay had a standard deviation of about $3 \mathrm{~ms}$. They also reported a significant difference in the performances of the various mouse models (or even of different versions of the same model).

Unfortunately, all these results deal only with the button timing for using a mouse as low-cost response box equipment, and may only serve to provide an indication of the usefulness of using the mouse as a velocity signal acquisition device. This is because the importance of the impact of movement recording on the timing characteristics of the mouse has not been properly addressed. Moreover, these studies recommended disabling the movement recording apparatus (e.g., removing the ball of a mechanical mouse) to ensure accurate acquisition of mouse button events.

Studies evaluating the precision of mouse motion tracking

A wealth of information on optical mouse acquisition characterization has been published in (Minoni \& Signorini, 2006; Ng, 2003; Ng \& Ang, 2004). For example, a linearity error of $0.1 \%$ for slow movements of at least $50 \mathrm{~mm}$ in amplitude has been reported in (Minoni \& Signorini, 2006). This seems more than acceptable for most human movement experiments. Minoni \& Signorini have also pointed out the significant influence of the working surface over acquisition accuracy, and reported good performances up to a speed of $380 \mathrm{~mm} / \mathrm{s}$, which is consistent with the optical sensor specifications.

\section{Characterization of computer mouse acquisition}

Although some information on sensor accuracy and on mouse timing precision is available, as shown in the present literature overview, this information is too scarce and incomplete to confidently use the computer mouse for acquisition in biomedical or psychological studies that focus on the characteristics of human movement velocity. The computer mouse has been designed as a pointing device, and so, for such an application, it would need good spatial accuracy, but not necessarily accurate timing. However, for studies dealing with the waveform of a movement, temporal precision is required.

Figure 1 shows a schema of the movement information flow, separating this process into four steps, each of which adds a given amount of noise to the ideal movement planned by a subject. Noise ${ }_{1}$ is both psychological and physiological. It conceptually encompasses everything that produces variability between the ideal movement the subject wants to perform and the real movement that is produced.

Noise $_{4}$ is specific to the modeling process. When aiming to use movement analysis for particular applications (e.g., in rehabilitation (Dipietro et al., 2009; O'Reilly \& Plamondon, 2009; Rohrer et al., 2004) or in stroke prevention (O'Reilly \& Plamondon, 2010; O'Reilly et al., 2009)), it is often more fruitful to use a human movement model (e.g., the minimization models (Engelbrecht, 2001), the equilibrium point models (Feldman \& Latash, 2005), the Kinematic

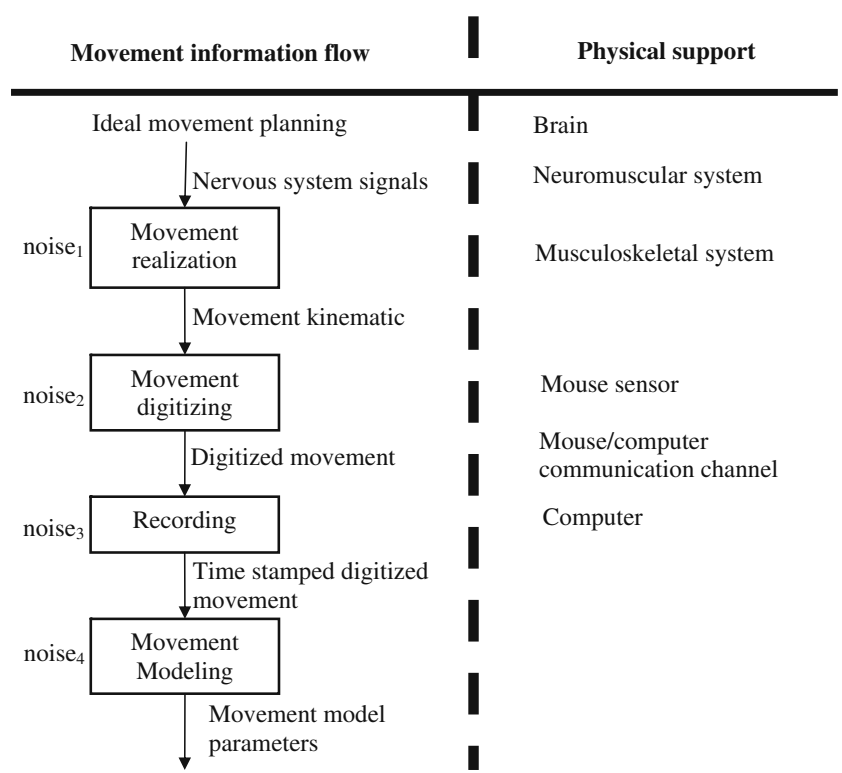

Fig. 1 Schema of the movement information flow for movement acquisition with a computer mouse 
Theory with its Delta-Lognormal model (Plamondon, 1995) and its Sigma-Lognormal model (Plamondon \& Djioua, 2006)). The parameters of these models are generally obtained to fit the real movement data as closely as possible. However, in practice, a perfect fit is never obtained, and so the values of the parameters that are used in statistical analysis contain a modeling noise, which is denoted here as noise ${ }_{4}$.

The impact of noise ${ }_{1}$ and noise ${ }_{4}$ are independent of the acquisition device, and so analysis of these noises is outside the scope of this study.

Most of the information available in the literature concerns the precision of optical sensor acquisition (Minoni \& Signorini, 2006; Ng, 2003; Ng \& Ang, 2004; Plant et al., 2003) and the delay of the mouse's internal circuitry (Beringer, 1992; Crosbie, 1990; Plant et al., 2003; Segalowitz \& Graves, 1990). This kind of information is useful for the characterization of noise 2 . It is complete enough, for example, for systems interfaced with an optical mouse sensor equipped with an appropriate acquisition card. In this scheme, only a negligible time delay is added between the movement digitization and its recoding (i.e., its time stamping). Therefore, in this case, virtually no noise is added at the third step of Fig. 1 (i.e., noise ${ }_{3} \approx \mathbf{0}$ ). This is also why most human motion tracking devices using an acquisition card (e.g., the VICON infra-red camera system) or including an embedded time stamping circuitry (e.g., Wacom digitizing tablets) remain virtually unaffected by deformation induced by a random time stamping delay.

However, unlike those systems, mouse signal acquisition on a Windows platform is not a real-time process, since it relies on a probability-based operating system with variable time delays. These are dependent on the system load and the priority of the various open processes. Therefore, a deformation of the time axis at step 3 (i.e., a possibly significant noise ${ }_{3}$ ) may be induced due to a random time delay between the movement digitization by the mouse and its time stamping by the computer. The importance of this deformation is for the most part unknown, and so it is unclear whether such a system would be adequate for human kinematic analysis. This is why the experiment and the data analysis presented in the next section are mainly concerned with the effect of noise $_{3}$ on the accuracy of human movement kinematic signals acquired with a computer mouse.

\section{Experimentation}

Hardware and software setup

Simultaneous acquisition with three systems

Every movement acquisition presented in this study has been performed simultaneously by three different sys-

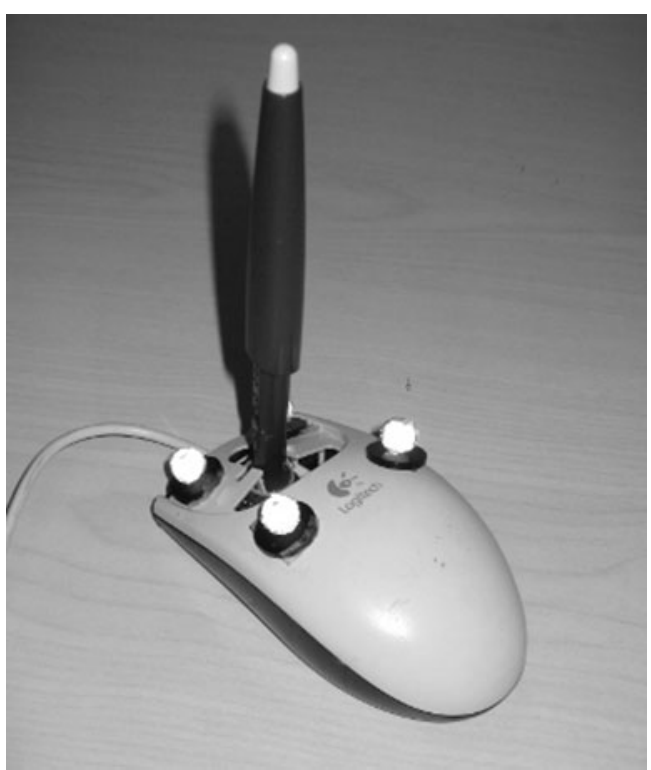

Fig. 2 Picture of the mouse modified to be tracked by the VICON system and the Wacom tablet

tems: a Logitech Optical USB mouse (model M-BJ58), a Wacom Intuos2 digitizing tablet, and a six infrared camera system (the VICON MX system with MX13 cameras). The simultaneous acquisition by these three systems has been achieved by placing four VICON markers on the mouse and fixing a Wacom pen through the mouse. To accomplish this, the scrolling wheel and the mouse button hardware had to be removed. No other changes were made to the mouse's functionality. Figure 2 shows the modified mouse.

\section{The VICON system}

The VICON ${ }^{3}$ MX13 camera signals are acquired in real time by an acquisition card. This means that their quality should not be affected by the computer's configuration. Data recorded by this system are the absolute position (relative to a fixed origin specified during the calibration of the system) of the markers in a 3D Cartesian space. In this study, a $200-\mathrm{Hz}$ sampling frequency was used.

This system has been widely and reliably used for biomechanical analysis of human movements, and it is therefore considered the gold standard in the present comparative study. Its signals have been employed as a benchmark to compare the tablet and the mouse performances and to calculate their signal-to-noise ratio (SNR). Data from the VICON MX system were sent to and saved by Computer 1 (see Table 1 for its main specifications).

\footnotetext{
$\overline{{ }^{3} \text { VICON: } 7388}$ S. Revere Parkway Suite 901, Centennial, CO 80112, USA, http://www.vicon.com
} 


\section{The Wacom tablet}

Data from the Wacom ${ }^{4}$ digitizers are acquired at $200 \mathrm{~Hz}$, and, most importantly, they are time stamped by embedded circuitry before being transmitted to the host computer via a USB port. This architecture ensures that their acquisition is independent of the specifications or the load of the host system. The data from the Wacom systems are absolute 2D Cartesian positions (relative to an origin fixed at the lower left-hand corner of the tablet). They were sent to and saved by Computer 1 .

\section{The computer mouse}

Unlike the VICON and Wacom systems, the timing of the acquisition with a USB mouse is not independent of its host computer. This is because the mouse does not time stamp its movement samples before sending them via the USB port. Therefore, the time stamping has to be performed by the host computer, which performs this task at a frequency that varies depending on many factors. For example, it may be influenced by the code of the program used for reading and time stamping the mouse information, as well as the availability of the host computer resources. As opposed to the VICON and the Wacom systems, the USB mouse sends information regarding positional change rather than absolute position. These data are in a 2D Cartesian space.

To ensure the applicability of our results to most computer mice, the model used for these tests has been deliberately chosen not to be a state-of-the-art pointing device. It is a low-cost (about \$20 US), relatively old mouse of average quality, such as those used on most personal computers. Unfortunately, virtually no technical information was available on this model, apart from the fact that its sensor has an 800 DPI resolution. No datasheet was available online, and Logitech was not ready to disclose any "Private and Confidential Proprietary Information" on the device.

For this experiment, the standard Windows XP driver for this mouse model was used, and the mouse signals were recorded using the Microsoft DirectInput library in $\mathrm{C}++.{ }^{5}$ We were interested in using this library primarily because, unlike a function such as GetCursorPos (...) from the Win32 API, DirectInput is not concerned with the mouse cursor position. Instead, it records the change in physical mouse position without interference of the mapping relation between the mouse movements and the screen cursor. The acquisition program pooled the mouse data using a

\footnotetext{
${ }^{4}$ Wacom Technology Corporation: 1311 SE Cardinal Court, Vancouver, WA 98683, http://www.wacom.com/index2.php

${ }^{5}$ The acquisition code may be obtained from the corresponding author.
}

multimedia timer with a 1-ms period. The collected data were time-stamped with the value of the Windows precision counter at the moment of pooling.

The mouse movements were recorded by Computer 2, the specifications of which are listed in Table 1. On this computer, the default Windows XP sampling frequency of $125 \mathrm{~Hz}$ for the USB controller used by the mouse was raised to $500 \mathrm{~Hz}$, using a procedure available on the Internet (Gamers With Jobs, 2009).

\section{Characteristics of the analyzed movement}

In this experiment, every recorded movement consisted of $30 \mathrm{~s}$ of an oscillatory motion performed by a subject ${ }^{6}$ on a guiding sheet (see Fig. 3) taped on a Wacom tablet. An oscillation tempo was given by a 50-ms "bip" sound occurring at a given frequency. Acquisitions were performed at nine different frequencies, with periods ranging from $100 \mathrm{~ms}$ to $500 \mathrm{~ms}$ in $50-\mathrm{ms}$ increments. There were three repetitions, for a total of 27 trials.

\section{Data manipulation and acquisition quality assessment}

The first and last two seconds of every trial were cut off and the remaining $26 \mathrm{~s}$ were used for computation. Position signals from the various systems were resampled at $1,000 \mathrm{~Hz}$ using a linear interpolation. Then, they were low-pass filtered at $25 \mathrm{~Hz}$ and differentiated to obtain the Cartesian velocity, which was used to compute the tangential speed using the Euclidean norm, that is: $v_{t}(t)=\sqrt{v_{x}^{2}(t)+v_{y}^{2}(t)+v_{z}^{2}(t)} \cdot$

The acquisition quality was assessed using the signal-tonoise ratio (SNR) defined in Eq. 1:

$S N R=10 \log \left(\frac{\int v_{t r}^{2}(t) d t}{\int\left(v_{t r}(t)-v_{t t}(t)\right)^{2} d t}\right)$

In this equation, $\mathrm{v}_{\mathrm{tr}}$ refers to the tangential velocity of the reference trajectory and $\mathrm{v}_{\mathrm{tt}}$ to the tangential velocity of the device tested. To allow the comparison of signals from the various devices, the $\mathrm{v}_{\mathrm{tt}}$ were time-aligned to the $\mathrm{v}_{\mathrm{tr}}$ by maximizing their cross-correlation. In turn, the signal for

\footnotetext{
${ }^{6}$ Every acquisition for this experiment was performed by only one subject, because the evaluation of neither the human motor control nor the physiological noise (i.e., noise ${ }_{1}$ ) is within the scope of this study. As only the mouse acquisition properties were studied, a mechanical actuator could have been used equally well. However, for convenience and simplicity, a human subject was preferred. This choice also ensures that the limitations of the mouse can be studied with natural humanoid motions.

${ }^{7}$ For the mouse and the Wacom system, $\mathrm{v}_{\mathrm{z}}(\mathrm{t})=0$, since these systems record planar movements. The $\mathrm{v}_{\mathrm{z}}$ component is included in this equation because the data from the VICON systems are threedimensional.
} 
Table 1 Principal characteristics of the computers used for data acquisition

\begin{tabular}{lll}
\hline Spec. & Computer 1 & Computer 2 \\
\hline OS & MS Windows XP Pro. Version 2002 & MS Windows XP Media Center Edition \\
& Service Pack 2 & Version 2002 \\
& & Service Pack 2 \\
CPU & Xeon $(\mathrm{TM})-3.00 \mathrm{GHz}$ & Intel Core2 CPU $-1.83 \mathrm{GHz}$ \\
RAM & $1 \mathrm{~GB}$ & $1 \mathrm{~GB}$ \\
\hline
\end{tabular}

each of the four VICON markers was used as a reference to compare the trajectory of the other three VICON markers, the mouse signal, and the tablet data.

A frequency correction was needed for the Wacom data, because the tablet's internal frequency was a little off the specified $200 \mathrm{~Hz}$, which caused a time compression of the signal that had a significant impact when comparing signals, given that these signals were $26 \mathrm{~s}$ long. We obtained an average frequency of $199.7949 \mathrm{~Hz}$ with a standard deviation of $0.0094 \mathrm{~Hz}$. The actual frequency was computed by comparing the duration of the acquisitions, as reported by the Wacom tablet, to the duration, as reported by the precision counter of the host computer. The 27 acquisitions (of approximately $30 \mathrm{~s}$ each) were used for this computation.

Since the various sensors and VICON markers are not located exactly at the same place, some precautions must be taken in comparing their trajectories. If no rotation of the mouse is allowed, the velocity of every sensor is identical. However, when the experiment described above is performed by a human subject, the oscillatory trajectory is generally curved and the mouse tends to be oriented along the radius of the resulting arc, inducing a small mouse rotation. Therefore, for example, the upper right-hand VICON marker consistently records a longer trajectory than the lower right-hand marker (see Fig. 4). To correct that movement-amplitude difference between the sensors, their trajectories were normalized in such a way that they were all of the same length. This was achieved by dividing the speed signal of each trajectory by its mean value and then multiplying that figure by the mean value of a reference speed signal, taken here as the speed of the lower right-hand VICON marker.

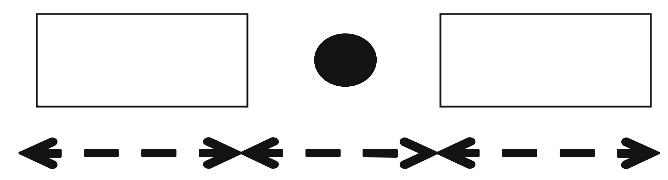

$50 \mathrm{~mm}$

$50 \mathrm{~mm}$

$50 \mathrm{~mm}$

Fig. 3 Pattern used to guide the subject's oscillations. Movements start from the black circle and then oscillate between the two rectangular zones at a pacing rate specified by a beeper

\section{Experimental results}

Figure 5 shows the results obtained using the experimental protocol and the data processing described above. On this graph, the lines represent the averaged SNR, and the whiskers show their $95 \%$ confidence intervals. These statistics were computed using all the repetitions and all the VICON markers as references. As an example, at a given oscillation frequency, the trajectory recorded by the Wacom tablet was compared to those of the four VICON markers and for each of the three repetitions. Therefore, 12 SNR samples were available for statistical computation in that case.

The frequency of the oscillatory movement was varied to study the effect of the movement speed on the acquisition accuracy. As can be observed on the graph, the mouse does not behave very well at high speed (i.e., in this specific case, for oscillation periods of less than $0.2 \mathrm{~s}$ ). However, it shows surprisingly good performances on slower movements. The non-real-time nature of the mouse acquisition does not seem to alter the data accuracy significantly compared to real-time acquisition devices (i.e., the Wacom tablet and the VICON system). Therefore, it seems that the use of the mouse as an acquisition device for planar human upper limb motion is limited by the maximum tracking speed of the optical sensor, rather than by the non-real-time nature of this device acquisition process. That is, the importance of noise 3 in Fig. 1 is negligible compared to the importance of noise $e_{2}$.

Figure 6 displays the 25th, 50th, 95th, and 100th percentiles of the speed distribution of the recorded movement as a function of the oscillation period. The data

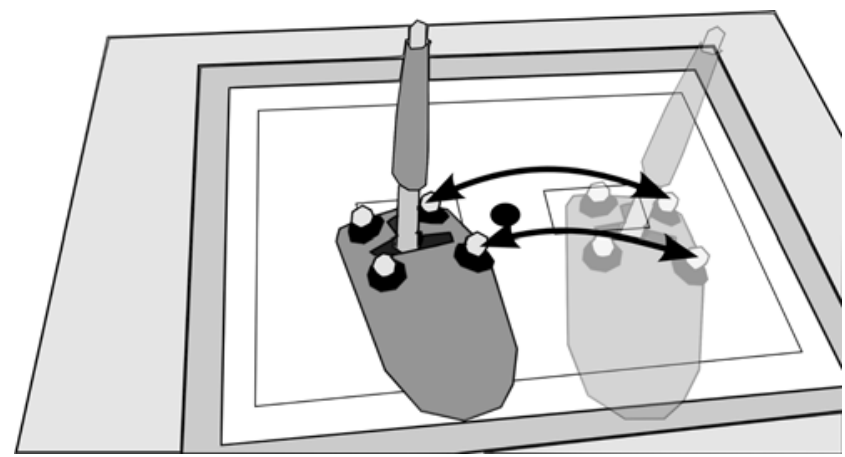

Fig. 4 Due to mouse rotation, the upper right-hand marker describes a larger oscillatory motion than the lower right-hand one 


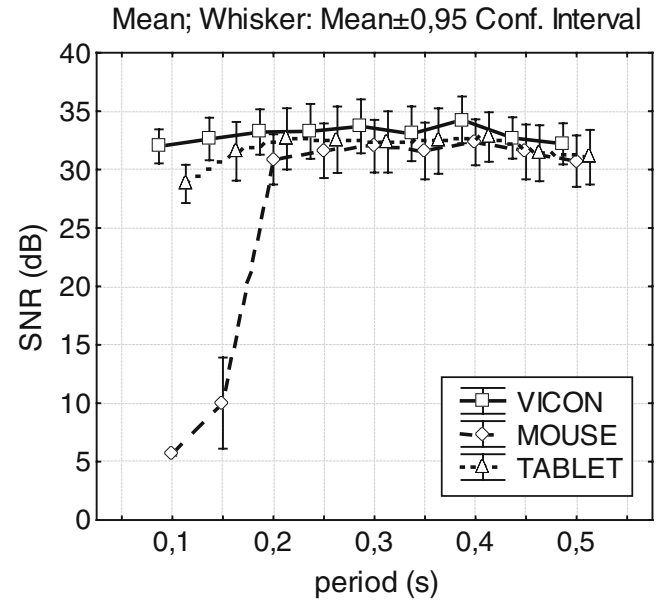

Fig. 5 SNR obtained for each acquisition device for different periods of oscillation

collected in our experiment show that the mouse is adequate for movement speed acquisition, even when movement velocity reaches $900 \mathrm{~mm} / \mathrm{s}$ (i.e., the maximum recorded speed for an oscillatory period of $0.2 \mathrm{~s}$ ). For velocity acquisition, this mouse model $^{8}$ can therefore be reliably used at a speed that is quite a bit higher than the $380 \mathrm{~mm} / \mathrm{s}$ recommended in (Minoni \& Signorini, 2006) for position acquisition.

\section{Discussion and recommendations}

General comments

It has been reported in (Djioua \& Plamondon, 2009a) that an SNR of $20 \mathrm{~dB}$ is generally enough to analyze kinematic signals in human movement research. Therefore, a computer mouse would seem to be a valid acquisition device for such studies.

This device is nevertheless more vulnerable to measurement error due to random time delays in movement recording, which may be induced by some greedy computer component drivers. It is also more likely to generate false readings if the subject inadvertently lifts it. For these reasons, experiments using a computer mouse as a human movement tracking device may benefit from collecting more specimens than experiments that rely on a more robust acquisition system. In this way, supplementary data will be available to detect and remove outlier recordings more easily.

The reader should be aware that, in our setup, there was no feedback from a computer screen. The mouse served only for movement acquisition, not for interacting with a software graphical user interface. To that end, we used the DirectInput library to record the physical mouse displacements directly,

\footnotetext{
${ }^{8}$ Unpublished experimental results also show similar performances for the Microsoft USB Wheel Mouse Optical.
}

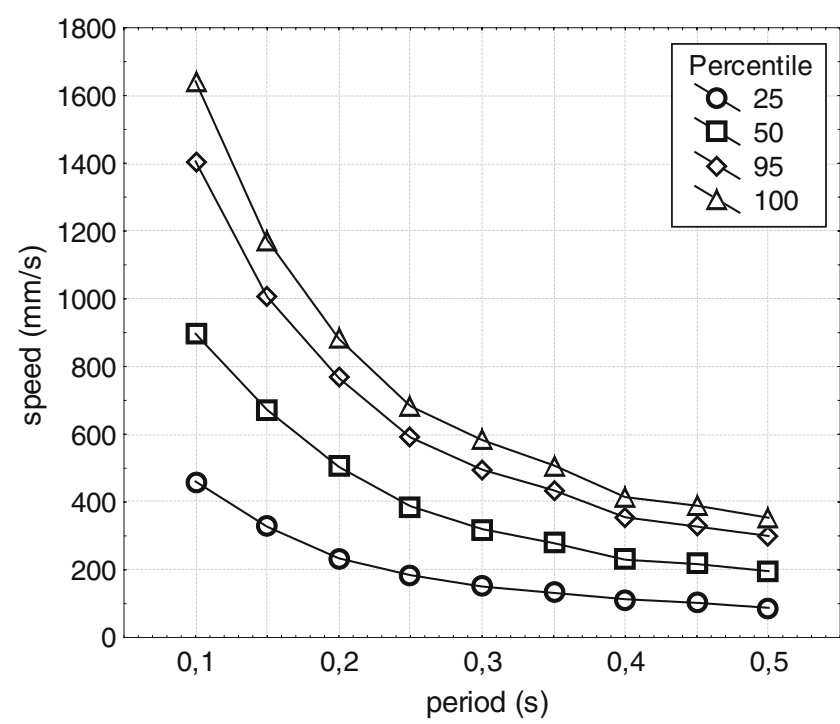

Fig. 6 This graph shows the 25th, 50th, 95th, and 100th percentiles of the speed distribution as taken from the lower right-hand VICON marker, averaged over the three repetitions. That is, the uppermost curve represents the maximal speed achieved by the mouse and the curve with the square markers indicates the median speed

rather than the movement of a cursor. Therefore, we did not use the mapping that the operating system applies to the mouse kinematics to render the cursor position. Experimenters interested in using a computer screen as a feedback device would have to take into account the delays associated with the display of information on a monitor. They would also have to consider the properties of the operating system cursor mapping (i.e., the mouse gain) or bypass it by appropriate low-level programming.

Limitation of this study

This study principally focused on investigating whether or not the probabilistic nature of Windows-based computer mouse acquisition was by itself a major limitation to using these motion-tracking devices in the human movement sciences. In doing so, the length of the recorded trajectories was normalized. This procedure does not allow for the evaluation of a potential systematic error on the gain value of the sensor. However, this is not necessarily a significant shortcoming for two reasons. First, a simple calibration procedure may be performed if needed. ${ }^{9}$ Second, for studies where there is more interest in the waveform of a movement than in its absolute amplitude (see (Fradet, Lee, \& Dounskaia, 2008) for an example of such a study), a minor systematic bias on the sensor gain may be irrelevant.

\footnotetext{
${ }^{9}$ For example, a few movements may be performed along a ruler to compare the distance recorded by the mouse to the distance measured on the ruler.
} 
Test design limitation

Until further examination, the velocity of almost straight trajectories (e.g., target reaching movements) should not be compared to the velocity of strongly curved trajectories (e.g., circle arc movements). Similarly, the velocity of straight trajectories oriented at $45^{\circ}$ with respect to the mouse's natural axis system should not be compared with the velocity of straight trajectories oriented near $90^{\circ}$ or $0^{\circ}$. These precautions are recommended to be consistent with (Palacin et al., 2006), who noted that the displacement error was maximal at $45^{\circ}$ and that a different calibration was needed for arc movements as compared to straight trajectories. This means that any type of movement (i.e., x-direction, $45^{\circ}$-direction, curved, straight) may be considered in a given experiment, but the results from different types of movement should not be compared without careful calibration of the mouse for each type of movement. This calibration should allow the estimation of the measurement standard error for each movement type. Ultimately, a statistical test may be used to verify whether the hypothesis of equality of these measurement errors is to be rejected.

Precautions should also be taken to control the working surface in the experimental design. As (Minoni \& Signorini 2006) pointed out, it can affect the recording of movement. They reported that, for optical mice, white paper gives good results, whereas surfaces that are black or have small white and black strips or similar patterns on them should be avoided.

The experimental protocol should be designed to obtain a movement speed slower than $900 \mathrm{~mm} / \mathrm{s}$. Such limitations can be implemented easily by imposing timing on the subject, by asking him to move at moderate speed, or by designing a task that requires enough spatial precision that sufficiently slow movements will be obtained without fail. ${ }^{10}$

As specified previously, this paper focuses on determining the adequacy of mouse acquisition for the analysis of movement velocity, with particular attention to the impact of the noise induced by the probabilistic nature of the mouse sampling in non-real-time systems, such as the Windows operating system (i.e., the noise ${ }_{3}$ in Fig. 1). The conclusions presented here may not be transferable to studies dealing with precise high-speed positional movement tracking, since the computer mouse is vulnerable to positional error accumulation. This is inherent to its movement-recording mode based on position changes, rather than on Cartesian positions with respect to a fixed origin. However, previous publications seem to indicate no problems in this regard if the displacement is relatively slow (Minoni \& Signorini, 2006; Ng, 2003).

\footnotetext{
${ }^{10}$ This is due to the natural speed-accuracy tradeoff in human motor control. See (R. Plamondon \& Alimi, 1997) for more information on this topic.
}

Mouse testing and calibration

To remain on the safe side, and until more studies have been conducted using a computer mouse, we suggest systematically performing some tests on using a mouse for data acquisition before using it in actual trials. Such tests should be used for calibration and for validating the consistency and accuracy of the acquisition procedure. They should be performed using the same setup (i.e., the same working surface, acquisition computer, acquisition software, mouse model, type of movements, etc.) as in the trials. These tests can be performed using a similar experimental protocol to the one presented here, but it could be simplified by testing only the movement performed in the planned trials (e.g., there is, of course, no need to repeat the acquisition procedure for various movement speeds if such variation is not expected in the trials) and by using the Wacom tablet as the gold standard. This device is quite a bit cheaper and easier to operate than a marker and camera system, such as the one used here, and it is capable of tracking mouse motion with a high degree of precision.

If the absolute gain of the mouse sensor also needs to be calibrated, we recommend doing so in a separate procedure and using a guide to restrain the mouse rotation. The two mouse axes should be calibrated independently.

\section{Conclusions}

This paper makes three principal contributions. First, with some conditions, it concludes that the computer mouse is reliable enough to be considered as an acquisition device for the analysis of human movement velocity signals.

Second, it shows that a mouse can be used in such a way that the impact of the probabilistic mouse-sampling process in a non-real-time operating system is not a key factor in the quality of the velocity signals gathered. Therefore, future investigations should focus on the quality of the mouse sensors (i.e., noise 2 ), rather than on the impact of the Windows operating system on the accuracy of the mouse acquisition process (i.e., noise ${ }_{3}$ ).

Third, it describes a methodology that can be used to test and calibrate a computer mouse using an affordable and user-friendly digitizing tablet. These results should apply to any type of study using a computer mouse, as long as its experimental protocol respects the restriction discussed in Sect. "Discussion and recommendations" and that its primary variable of interest is the velocity of an end effector.

To conclude, either a computer mouse or a tablet can be used for the acquisition of planar human movements, depending on the context. Tablets are preferable in an 
experimental context where the accuracy of the movement is of paramount importance, where cost is of secondary importance, and where a single apparatus can be used by many subjects (i.e., one tablet for $\mathrm{X}$ subjects). However, it may be preferable to consider mouse acquisition when utilization of the acquisition device will be widely distributed (i.e., $\mathrm{X}$ mice for $\mathrm{X}$ subjects), when the low cost and ubiquity of the acquisition device is highly important, and when supplementary acquisition can be recorded to compensate for the lower confidence level associated with mouse acquisition. These would be the requirements in the case of at-home longitudinal experiments, in telemedicine, and potentially in Web-based applications, for example. For such applications, a single tablet could be used early on in the project to calibrate a large number of mice. Analysis of the results from those calibrations should reveal whether or not the calibration process is necessary in subsequent phases of the project.

Acknowledgments This work was partly supported by NSERC grant RGPIN-915 to Dr. Réjean Plamondon. The VICON MX system was purchased with CFI grant 2959 to Dr. Mohamad Sawan, principal investigator of a research team that included Dr. Plamondon.

\section{References}

Ahmed, A. A. E., \& Traore, I. (2007). A new biometric technology based on mouse dynamics. IEEE Transactions on Dependable and Secure Computing, 4(3), 165-179.

Beringer, J. (1992). Timing accuracy of mouse response registration on the IBM microcomputer family. Behavior Research Methods, Instruments, \& Computers, 24(3), 486-490.

Biryukova, E. V., Roby-Brami, A., Frolov, A. A., \& Mokhtari, M. (2000). Kinematics of human arm reconstructed from spatial tracking system recordings. Journal of Biomechanics, 33(8), 985-995.

Bonarini, A., Matteucci, M., \& Restelli, M. (2005). Automatic Error Detection and Reduction for an Odometric Sensor based on Two Optical Mice. Paper presented at the 2005 IEEE International Conference on Robotics and Automation, and published in the Proceedings of this conference.

Brenner, E., \& Smeets, J. B. J. (2003). Fast corrections of movements with a computer mouse. Spatial Vision, 16(3-4), $365-376$.

Cooney, J. A., Xu, W. L., \& Bright, G. (2004). Visual dead-reckoning for motion control of a Mecanum-wheeled mobile robot. IEEE/ ASME Transactions on Mechatronics, 14(6), 623-637.

Crosbie, J. (1990). The microsoft mouse as a multipurpose response device for the IBM-PC/XT/AT. Behavior Research Methods, Instruments, \& Computers, 22(3), 305-316.

Daya, J., Jinghua, X., Haihong, L., \& Qionglin, D. (2007). New approaches to data acquisition in a torsion pendulum experiment. European Journal of Physics, 28(5), 977-982.

Dipietro, L., Krebs, H. I., Fasoli, S. E., Volpe, B. T., \& Hogan, N. (2009). Submovement changes characterize generalization of motor recovery after stroke. Cortex, 45(3), 318-324.

Djioua, M., \& Plamondon, R. (2009a). A new algorithm and system for the characterization of handwriting strokes with deltalognormal parameters. IEEE Transactions on Pattern Analysis and Machine Intelligence, 31(11), 2060-2072.
Djioua, M., \& Plamondon, R. (2009b). Studying the variability of handwriting patterns using the kinematic theory. Human Movement Science, 28(5), 588-601.

Ehara, Y., Fujimoto, H., Miyazaki, S., Mochimaru, M., Tanaka, S., \& Yamamoto, S. (1997). Comparison of the performance of 3D camera systems II. Gait \& Posture, 5(3), 251-255.

Ehara, Y., Fujimoto, H., Miyazaki, S., Tanaka, S., \& Yamamoto, S. (1995). Comparison of the performance of 3D camera systems. Gait \& Posture, 3(3), 166-169.

Engelbrecht, S. E. (2001). Minimum principles in motor control. Journal of Mathematical Psychology, 45(3), 497-542.

Everaert, D. G., Spaepen, A. J., Wouters, M. J., Stappaerts, K. H., \& Oostendorp, R. A. (1999). Measuring small linear displacements with a three-dimensional video motion analysis system: Determining its accuracy and precision. Archives of Physical Medicine and Rehabilitation, 80(9), 1082-1089.

Faustina, H., Simeon, K., Patrick, L., \& John, C. (2004). Mouse movements of motion-impaired users: A submovement analysis. SIGACCESS Access Comput, 77-78, 102-109.

Feldman, A. G., \& Latash, M. L. (2005). Testing hypotheses and the advancement of science: Recent attempts to falsify the equilibrium point hypothesis. Experimental Brain Research, 161(1), 91-103.

Fradet, L., Lee, G., \& Dounskaia, N. (2008). Origins of submovements in movements of elderly adults. Journal of Neuroengineering and Rehabilitation, 5(1), 28-41.

Gilboa, Y., Josman, N., Fattal-Valevski, A., Toledano-Alhadef, H., \& Rosenblum, S. (2010). The handwriting performance of children with NF1. Research in Developmental Disabilities, 31(4), 929-935.

Hassan, E. A., Jenkyn, T. R., \& Dunning, C. E. (2007). Direct comparison of kinematic data collected using an electromagnetic tracking system versus a digital optical system. Journal of Biomechanics, 40(4), 930-935.

Impedovo, D., \& Pirlo, G. (2008). Automatic signature verification: The state of the art. IEEE Transactions on Systems, Man, and Cybernetics. Part C: Applications and Reviews, 38(5), 609-635.

Jansen, A. R., Blackwell, A. F., \& Marriott, K. I. M. (2003). A tool for tracking visual attention: The restricted focus viewer. Behavior Research Methods, Instruments, \& Computers, 35(1), 57-69.

Kisacanin, B., Agarwal, G. C., Taber, J., \& Hier, D. (2000). Computerised evaluation of cognitive and motor function. Medical \& Biological Engineering \& Computing, 38(1), 68-73.

Kok-Meng, L., \& Debao, Z. (2004). A real-time optical sensor for simultaneous measurement of three-DOF motions. IEEE/ASME Transactions on Mechatronics, 9(3), 499-507.

Kolahi, A., Hoviattalab, M., Rezaeian, T., Alizadeh, M., Bostan, M., \& Mokhtarzadeh, H. (2007). Design of a marker-based human motion tracking system. Biomedical Signal Processing and Control, 2(1), 59-67.

Lange, K. W., Tucha, L., Walitza, S., Gerlach, M., Linder, M., \& Tucha, O. (2007). Interaction of attention and graphomotor functions in children with attention deficit hyperactivity disorder. Journal of Neural Transmission. Supplementum, 72, 249-259.

Leclerc, F., \& Plamondon, R. (1994). Automatic signature verification: The state of the art 1989-1993. International Journal of Pattern Recognition and Artificial Intelligence, 8(3), 643-659.

Mayagoitia, R. E., Nene, A. V., \& Veltink, P. H. (2002). Accelerometer and rate gyroscope measurement of kinematics: An inexpensive alternative to optical motion analysis systems. Journal of Biomechanics, 35(4), 537-542.

Mills, P. M., Morrison, S., Lloyd, D. G., \& Barrett, R. S. (2007). Repeatability of 3D gait kinematics obtained from an electromagnetic tracking system during treadmill locomotion. Journal of Biomechanics, 40(7), 1504-1511.

Minoni, U., \& Signorini, A. (2006). Low-cost optical motion sensors: An experimental characterization. Sensors and Actuators A: Physical, 128(2), 402-408. 
Morein-Zamir, S., Chua, R., Franks, I., Nagelkerke, P., \& Kingstone, A. (2006). Measuring online volitional response control with a continuous tracking task. Behavior Research Methods, 38(4), 638-647.

Nagel, F., Kopiez, R., Grewe, O., \& Altenmuller, E. (2007). EMuJoy: Software for continuous measurement of perceived emotions in music. Behavior Research Methods, 39(2), 283-290.

$\mathrm{Ng}$, T. W. (2003). The optical mouse as a two-dimensional displacement sensor. Sensors and Actuators A: Physical, 107 (1), 21-25.

Ng, T. W., \& Ang, K. T. (2004). The optical mouse for vibratory motion sensing. Sensors and Actuators A: Physical, 116(2), 205-208.

Ng, T. W., \& Ang, K. T. (2005). The optical mouse for harmonic oscillator experimentation. American Journal of Physics, 73(8), 793-795.

Ng, T. W., \& Carne, M. (2007). Optical mouse digital speckle correlation. Optics Communications, 280(2), 435-437.

Ng, T. W., \& Cheong, T. L. (2004). The optical mouse as an inexpensive region-of-interest position recorder in optical microscopy. Microscopy Research and Technique, 63(4), 203-205.

Nijhof, E.-J. (2003). On-line trajectory modifications of planar, goaldirected arm movements. Human Movement Science, 22(1), 13-36.

O'Reilly, C., \& Plamondon, R. (2009). A lognormal framework for human movement rehabilitation. In Y. K. Tan (Ed.), Rehabilitation engineering (pp. 157-172). Vukovar: IN-TECH.

O'Reilly, C., \& Plamondon, R. (2010). Impact of the principal stroke risk factors on human movements. Human Movement Science. doi:10.1016/j.humov.2010.07.010

O’Reilly, C., Plamondon, R., Lebrun, L.-H., Clément, B., \& Mathieu, P. A. (2009). Sigma-lognormal analysis of a complex movements neuromuscular test. Paper presented at the 14th Conference of the International Graphonomics Society, Dijon, France.

Ochoa, O. R., \& Kolp, N. F. (1997). The computer mouse as a data acquisition interface: Application to harmonic oscillators. American Journal of Physics, 65(11), 1115-1118.

Ogawa, K., Inui, T., \& Sugio, T. (2007). Neural correlates of state estimation in visually guided movements: An event-related fMRI study. Cortex, 43(3), 289-300.

Palacin, J., Valganon, I., \& Pernia, R. (2006). The optical mouse for indoor mobile robot odometry measurement. Sensors and Actuators A: Physical, 126(1), 141-147.

Phillips, J. G., \& Triggs, T. J. (2001). Characteristics of cursor trajectories controlled by the computer mouse. Ergonomics, 44 (5), 527-536.

Picerno, P., Cereatti, A., \& Cappozzo, A. (2008). Joint kinematics estimate using wearable inertial and magnetic sensing modules. Gait \& Posture, 28(4), 588-595.

Plamondon, A., Delisle, A., Larue, C., Brouillette, D., McFadden, D., Desjardins, P., et al. (2007). Evaluation of a hybrid system for three-dimensional measurement of trunk posture in motion. Applied Ergonomics, 38(6), 697-712.

Plamondon, R. (1995). A kinematic theory of rapid human movements. Part I. Movement representation and generation. Biological Cybernetics, 72(4), 295-307.

Plamondon, R., \& Alimi, A. M. (1997). Speed/accuracy trade-offs in target-directed movements. The Behavioral and Brain Sciences, 20(2), 279-303.

Plamondon, R., \& Djioua, M. (2006). A multi-level representation paradigm for handwriting stroke generation. Human Movement Science, 25(4-5), 586-607.

Plamondon, R., \& Lorette, G. (1989). Automatic signature verification and writer identification - the state of the art. Pattern Recognition, 22(2), 107-131.
Plamondon, R., \& Srihari, S. N. (2000). Online and offline handwriting recognition: A comprehensive survey. Pattern Analysis and Machine Intelligence, IEEE Transactions on, 22(1), 63-84.

Plant, R. R., Hammond, N., \& Whitehouse, T. (2003). How choice of mouse may affect response timing in psychological studies. Behavior Research Methods, Instruments, \& Computers, 35(2), 276-284.

Pratt, J., \& Turk-Browne, N. B. (2003). The attentional repulsion effect in perception and action. Experimental Brain Research, 152(3), 376-382.

Richards, J. G. (1999). The measurement of human motion: A comparison of commercially available systems. Human Movement Science, 18(5), 589-602.

Roby-Brami, A., \& Burnod, Y. (1995). Learning a new visuomotor transformation: Error correction and generalization. Brain Research. Cognitive Brain Research, 2(4), 229-242.

Rohrer, B., Fasoli, S., Krebs, H. I., Volpe, B., Frontera, W. R., Stein, J., et al. (2004). Submovements grow larger, fewer, and more blended during stroke recovery. Motor Control, 8(4), 472-483.

Santos, F., Silva, V., \& Almeida, L. (2002). A robust self-localization system for a small mobile autonomous robot. Paper presented at the Proc. of the IEEE Int. Symp. on Robotics and Automation.

Segalowitz, S. J., \& Graves, R. E. (1990). Suitability of the IBM-XT, $\mathrm{AT}$, and PS/2 keyboard, mouse, and game port as response devices in reaction-time paradigms. Behavior Research Methods, Instruments, \& Computers, 22(3), 283-289.

Singh, S. P. N., \& Waldron, K. J. (2004). Design and evaluation of an integrated planar localization method for desktop robotics. Paper presented at the 2004 IEEE International Conference on Robotics and Automation, and published in the Proceedings of this conference.

Slijper, H., Richter, J., Over, E., Smeets, J., \& Frens, M. (2009). Statistics predict kinematics of hand movements during everyday activity. Journal of Mot. Behavior, 41(1), 3-9.

Swett, B. A., Contreras-Vidal, J. L., Birn, R., \& Braun, A. (2010). Neural substrates of graphomotor sequence learning: A combined fMRI and kinematic study. Journal of Neurophysiology, 103(6), 3366-3377.

Thompson, S. G., McConnell, D. S., Slocum, J. S., \& Bohan, M. (2007). Kinematic analysis of multiple constraints on a pointing task. Human Movement Science, 26(1), 11-26.

Van Gemmert, A. W. A., Teulings, H.-L., \& Stelmach, G. E. (2001). Parkinsonian patients reduce their stroke size with increased processing demands. Brain and Cognition, 47(3), 504-512.

Walker, N., Meyer, D. E., \& Smelcer, J. B. (1993). Spatial and temporal characteristics of rapid cursor-positioning movements with electromechanical mice in human-computer interaction. Human Factors: The Journal of the Human Factors and Ergonomics Society, 35(3), 431-458.

Young, S. J., Pratt, J., \& Chau, T. (2008). Choosing the fastest movement: Perceiving speed-accuracy tradeoffs. Experimental Brain Research, 185(4), 681-688.

Zhou, H. Y., \& Hu, H. S. (2008). Human motion tracking for rehabilitation - a survey. Biomedical Signal Processing and Control, 3(1), 1-18.

Zhou, H., Hu, H., Harris, N. D., \& Hammerton, J. (2006). Applications of wearable inertial sensors in estimation of upper limb movements. Biomedical Signal Processing and Control, 1 (1), 22-32.

Zhou, H., Stone, T., Hu, H., \& Harris, N. (2008). Use of multiple wearable inertial sensors in upper limb motion tracking. Medical Engineering \& Physics, 30(1), 123-133. 